\title{
Relativistic Atomic Level Calculations with Nuclei Carrying Additional Fractional Charges
}

\author{
Khalid Rashid \\ Nuclear Physics Division, Pakistan Institute of Nuclear Science and Technology, \\ Nilore, Rawalpindi, Pakistan \\ Burkhard Fricke \\ Fachbereich Physik der Universität, Kassel, Federal Republic of Germany
}

Received December 28, 1982

One-electron energy levels and wavelengths have been calculated for Na-like ions whose nuclei carry quarks with additional charges $\pm e / 3, \pm 2 e / 3$. The calculations are based on relativistic self-consistent field procedures. The deviations from experimental values exhibit regularities which allow an extrapolation for the wavelengths of $3 s-3 p, 3 s-4 p$, $3 p-3 d$, and $3 p-4 s$ transitions for the nuclear charge $Z=11 \pm 1 / 3, \pm 2 / 3$.

A number of transitions are found in the region of visible light which could be used in an optical search for quark atoms.

\section{Introduction}

Although the present gauge theories of quarks lead to a local confinement model (in general) and do not allow for the existence of free quarks, experimental observations of fractional charges $(e / 3$ and $2 e / 3)$ on matter have been recently reported by La Rue et al. [1]. This has given rise to considerable speculation that atoms with fractionally charged quarks bound to nuclei may exist. If this is the case, the application of laser spectroscopic single atom detection methods may lead to experimental identification of quarks by observation of their atomic spectra [2].

The term "quark atom", used here, stands for an atom whose nucleus carries an additional fractional charge $\pm e / 3, \pm 2 e / 3$. The change in the nuclear charge causes significant shifts in the atomic energy levels, particularly for valence electron levels. If an optical search for such quark atoms is to be successful, it is essential to know the atomic spectra of these atoms theoretically with reasonable accuracy. In this paper an attempt is made to calculate the energy levels of such atoms from first principles. Theoretical methods, permitting directly very good quantitative predictions of term energies and transition wavelengths, are very difficult and too tedious, except for the lightest elements [3]. However, significant progress has been made in the development of Dirac-Fock atomic codes based on relativistic self-consistent field procedures
$[4,5]$. In this paper the Desclaux code $[5]$ was used to calculate the atomic levels of Na-like systems. In this work we shall restrict our attention to the valence electron levels of Na-like quark atoms, which were choosen for their relative simplicity of structure. There is only one valence electron surrounding the core of closed shell orbitals and thus we avoid the problems of open shell configurations. Because all configurations arising from inner shell excitations are higher than the series limit, the one-electron term system for this eleven-electron iso-electronic system will have a very smooth dependence on $Z$. This fact makes it possible to develope interpolation formulae from experimental data for such iso-electronic sequences. These formulae have been used very successfully for predicting transition wavelengths for highly ionized ions [6]. However, caution has to be exercised in using these interpolation formulae around the neutral atom $(Z=11)$. In particular towards the negative ion side $(Z=102 / 3$, $101 / 3$ ) the extrapolation formulae seem to be quite complicated.

The relativistic self-consistent field method yields quite accurate results for energy levels of inner shell electrons and also for valence electrons of highly charged ions. However, the accuracy for energy levels of the valence electrons drops for neutral or quasi neutral atoms, where, in our case, the relative deviations 
between the calculated and observed values can be as large as $6 \%$. However, as stated above, the relative deviations exhibit $Z$-dependent regularities in their behaviour. Using extrapolations for the differences between theoretical and experimental values we obtain a second estimate for the transition energies besides the values from the extrapolation formulae, which allow a relatively safe prediction of the optical transitions of "quark atoms" with $Z=11 \pm 1 / 3, \pm 2 / 3$.

\section{Method and Results}

The calculations are based on the relativistic selfconsistent field method. The details of the computer program, which calculates averaged total energies for electronic systems, are described in reference [5]. In addition the QED-effects vacuum polarisation and vacuum fluctuation are included in first-order perturbation theory. For the transitions under investigation here these effects are insignificant.

We performed calculations for different configurations defined by the valence electron levels. The transition energies are obtained in adiabatic approximation by building the differences between the total atomic energies of the two states involved. For all calculations the nucleus was assumed to have a uniform charge distribution of radius $1.2 \AA^{1 / 3} \mathrm{fm}$, where $A$ is the massnumber. However, the effect of the finite size of the nucleus in transition wavelength of valence electron is negligible. For example, for the $3 s-4 p$ transition it is of the order of $10^{-5} \AA$.

Table 1 gives the calculated and observed energy levels relative to the ground-state $(3 s$-level). The relative deviations $\Delta$ between the observed and calculated values, given in $\%$, are interpolated to the $Z=11 \pm 1 / 3$, $\pm 2 / 3$ cases. The $a b$-initio theoretical values are then corrected for these deviations. The corrected values $T h^{c}$ are also given in Table 1 . In Tables $2 \mathrm{a}-2 \mathrm{e}$ we have listed the theoretical and experimental values for the $\mathrm{Na}$ atom and for $\mathrm{Na}$-like ions for the $3 s-3 p, 3 s-3 d$, $3 s-4 p$, and $3 p-4 s$ transitions. The relative deviations $\delta$, in $\%$ of the theoretical values from the experimental values, are given in the first part of the third column. Naturally, $\delta$ values for experimentally observed cases, i.e. $Z=11,12,13$, are obtained by direct comparison. For non-integer $Z$ the $\delta$ values, given in the second part of column 3 , are derived by direct interpolation with the help of Lagrange formulae.

For comparison of relative accuracies we have included here the case of highly ionized Na-like $\mathrm{ZnXX}$, $Z=30$, ion. The deviations $\delta$ in general increase sys-

Table 1. Energy Levels of Na-like Systems for Z-values from $101 / 3$ to 13 . The experimental (Exp), theoretical (Th), and corrected theoretical $\left(\mathrm{Th}^{\mathrm{c}}\right)$ values are given in $\mathrm{cm}^{-1}$. The values are normalized to zero for the groundstate $(3 \mathrm{~s}) . \Delta$ is defined as 100 (Exp-Th)/Exp. The conversion factor used is: $\mathrm{leV}=8,065.7324 \mathrm{~cm}^{-1}$. Th ${ }^{c}$ are the theoretical corrected values which are obtained by correcting the $a b$-initio values for the deviation $\Delta$. The values of $\Delta$ for $Z=11 \pm 1 / 3, \pm 2 / 3$ are obtained by interpolation

\begin{tabular}{|c|c|c|c|c|c|c|c|}
\hline$Z$ & & $3 s_{1 / 2}$ & $3 p_{1 / 2}$ & $3 p_{3 / 2}$ & $3 d_{5 / 2}$ & $4 s_{1 / 2}$ & $4 p_{3 / 2}$ \\
\hline \multirow[t]{3}{*}{$10 \frac{1}{3}$} & Th & 0 & $3,811.45$ & $3,813.2724$ & $5,433.81$ & $4,574.87$ & $5,457.45$ \\
\hline & $\mathrm{Th}^{c}$ & 0 & $4,175.33$ & $4,177.40$ & $5,845.51$ & $4,887.94$ & $5,826.25$ \\
\hline & $\Delta$ & 0 & 8.7153 & 8.71666 & 7.043 & 6.405 & 6.330 \\
\hline \multirow[t]{3}{*}{$10 \frac{2}{3}$} & Th & 0 & $9,726.215$ & $9,731.790$ & $15,594.126$ & $13,414.603$ & $15,903.602$ \\
\hline & $\mathrm{Th}^{c}$ & 0 & $10,489.58$ & $10,489.55$ & $16,564.121$ & $14,178.09$ & $16,794.20$ \\
\hline & $\Delta$ & 0 & 7.2243 & 7.224 & 5.856 & 5.385 & 5.303 \\
\hline \multirow[t]{3}{*}{11} & Th & 0 & $15,964.50$ & $15,971.01$ & $27,767.53$ & $24,585,03$ & $28,341.98$ \\
\hline & Exp & 0 & $16,956.183$ & $16,973.38$ & $29,172.90$ & $25,739.86$ & $30,272.51$ \\
\hline & $\Delta$ & 0 & 5.9073 & 5.905 & 4.817 & 4.486 & 4.40 \\
\hline \multirow[t]{3}{*}{$11 \frac{1}{3}$} & Th & 0 & $22,207.90$ & $22,241.26$ & $41,074.70$ & $37,540.34$ & $43,909.84$ \\
\hline & $T h^{c}$ & 0 & $23,318.92$ & $23,353.34$ & $42,749.63$ & $38,986.34$ & $45,559.08$ \\
\hline & $\Delta$ & 0 & 4.1644 & 4.762 & 3.918 & 3.709 & 3.62 \\
\hline \multirow[t]{3}{*}{$11 \frac{2}{3}$} & Th & 0 & $28,431.07$ & $28,487.36$ & $55,097.02$ & $52,069.14$ & $60,543.81$ \\
\hline & $T h^{c}$ & 0 & $29,552.79$ & $29,610.48$ & $56,896.42$ & $53,708.87$ & $62,392.50$ \\
\hline & $\Delta$ & 0 & 3.7956 & 3.793 & 3.1626 & 3.053 & 2.963 \\
\hline \multirow[t]{3}{*}{12} & Th & 0 & $34,598.90$ & $35,686.73$ & $69,664.59$ & $68,045.93$ & $78,689.11$ \\
\hline & Exp & 0 & $35,669.31$ & $35,760.88$ & $71,491.06$ & $69,804.95$ & $80,650.02$ \\
\hline & $\Delta$ & 0 & 3.0009 & 3.000 & 2.55 & 2.52 & 2.43 \\
\hline \multirow[t]{3}{*}{13} & Th & 0 & $52,791.17$ & $53,018.66$ & $114,139.45$ & $124,080.36$ & $141,460.49$ \\
\hline & Exp & 0 & $53,682.93$ & $53,916.60$ & $115,958.50$ & $126,164.05$ & $143,713.50$ \\
\hline & $\Delta$ & 0 & 1.6612 & 1.67 & 1.57 & 1.65 & 1.57 \\
\hline
\end{tabular}


Tables 2. The a) $3 s-3 p_{1 / 2}$, b) $3 s-3 p_{3 / 2}$, c) $3 p-3 d_{5 / 2}$, d) $3 s-4 p_{3 / 2}$, and e) $3 p_{3 / 2}-4 s$ transitions for Na-like ions for different values of $Z$ from $101 / 3$ to 13 in $\AA$. The experimental values have been taken from Bashkin [7]

\begin{tabular}{lllllll}
\hline $\begin{array}{l}Z \text { of } \\
\text { Na-like } \\
\text { ions }\end{array}$ & $\begin{array}{l}\text { Theoretical } \\
(\AA)\end{array}$ & $\begin{array}{l}\text { Experimental } \\
(\AA)\end{array}$ & Differences (in \%) & & Predicted values \\
\cline { 5 - 6 } \cline { 5 - 6 } & & exp-theory & extrapolated & & $\delta$-corrected $\Delta$-corrected $\begin{array}{l}\text { extrapolated } \\
\text { (Edlen) }\end{array}$ \\
\hline
\end{tabular}

a. $3 s^{2} S_{1 / 2}-3 p^{2} P_{1 / 2}$ transitions

$10 \frac{1}{3} \quad 26,227$

$10 \frac{2}{3} \quad 10,276$

$11 \quad 6,262$

$11 \frac{1}{3} \quad 4,496$

$11 \frac{2}{3} \quad 3,510$

$12 \quad 2,883$

$\begin{array}{ll}12 & 2,883 \\ 13 & 1,886 \\ 30 & 255.94\end{array}$

$-9.366$

$-7.745$

23,980

23,939

26,253

$-6.316$

$-5.072$

9,537

9,534

$9,544.5$

$-4.016$

4,279

5,891

3,374

4,282

4,285

$\begin{array}{ll}2,795 & -3.148 \\ 1,855 & -1.671\end{array}$

3,377

3,379

2,796

1,855

0.57

257

b. $3 s^{2} S_{1 / 2}-3 p^{2} P_{3 / 2}$ transitions

256.406

$10 \frac{1}{3} \quad 26,226.46$

$10 \frac{2}{3} \quad 10,282.58$

$11 \quad 6,269.18$

$11 \frac{1}{3} \quad 4,502.84$

$11 \frac{2}{3} \quad 3,517.30$

$12 \quad 2,890.33$

$1,894.25$

$\begin{array}{rr}13 & 1,894.25 \\ 30 & 286.21\end{array}$

\section{5,896}

$2,802.7$

$1,862.79$

288.126

c. $3 p^{2} P_{3 / 2}-3 d^{2} D_{5 / 2}$ transitions

$10 \frac{1}{3} \quad 61,731$

$10 \frac{2}{3} \quad 17,059$

$11 \quad 8,476.7$

$11 \frac{1}{3} \quad 5,310$

$11 \frac{2}{3} \quad 3,758$

$12 \quad 2,867$

$13 \quad 1,636$

$30 \quad 209.95$

210.60

8,195

d. $3 s^{2} S_{1 / 2}-4 p^{2} P_{3 / 2}$ transitions

$\begin{array}{rr}10 \frac{1}{3} & 18,324 \\ 10 \frac{2}{3} & 6,288\end{array}$

$11 \quad 3,455$

$11 \frac{1}{3} \quad 2,278$

$11 \frac{2}{3} \quad 1,652$

$12 \quad 1,271$

$13 \quad 707$

$30 \quad 34.06$

696

696
34.0

3,302

e. $3 p^{2} P_{3 / 2}-4 s^{2} S_{1 / 2}$ transitions

$10 \frac{1}{3} \quad 131,314$

$10 \frac{2}{3} \quad 27,154$

$11 \quad 11,608$

$11 \frac{1}{3} \quad 6,537$

$11 \frac{2}{3} \quad 4,240$

$12 \quad 2,997$

$13 \quad 1,407$

$30 \quad 41.94$
$-0.03$

$34.0-0.03$

11,404

2,936
1,384
41.828

$-1.70$
$-9.445$

$-6.329$

$-7.789$

$-5.065$

$-3.998$

$-3.126$

$-1.689$

0.67

$-3.437$

$$
-4.126
$$$$
-3.778
$$

$-3.104$

$-2.778$

$-2.46$

$-1.552$

0.10

$-4.634$

$-6.726$

$$
-5.61
$$

$-3.787$

$-3.076$

$-2.50$

$-1.58$

$\begin{array}{ccc}23,963 & 23,951 & 26,266 \\ 9,539.52 & 9,539 & 9,550.46 \\ & & 5,897.8 \\ 4,285.73 & 4,288.53 & 4,292 \\ 3,382.08 & 3,384 & 3,386.45 \\ & & 2,803.64 \\ & & 1,862.92 \\ & & 287.33\end{array}$

$59,285 \quad 59,950$

$16,438 \quad 16,462$

$5,150 \quad 5,155.8$

$3,656.42 \quad 3,665$

140,743

27,112

6,397

4,150 
tematically for decreasing ionization. It is largest for neutral atoms. The variation in $\delta$ is very rapid from the single ionized case of $\mathrm{MgII}$ to the neutral atom case of $\mathrm{NaI}$. In the case of a fractional negative noninteger charge bound to the nucleus we have a negative Na-like atom. Assuming that the systematic increase of $\delta$ continues from the neutral NaI case to the negatively charged $\mathrm{Na}$ atom, we have extrapolated the ' $\delta$ values to $Z=102 / 3$ and $101 / 3$. For $Z=111 / 3$, and $112 / 3 \delta$ values are obtained by interpolation and therefore are much more reliable. Using the $\delta$ values of the second part of column 3 we get the predictions for the transition wavelengths in the first part of column 4 under " $\delta$-corrected". The second possibility to predict these wavelengths is to use the differences of the extrapolations of Term energies from Table 1. These results are given in column 4 under " $\Delta$-corrected". In general the $\delta$ values show a regular behaviour: They increase with decreasing $Z$ value. The only exception is the $3 p-4 s$ transition, here the $\delta$ value for the neutral $\mathrm{NaI}$ atom is smaller than that for the singly ionized $\mathrm{MgII}$. The reason for this inconsistency is not clear. Therefore, for this case we did not extrapolate the $\delta$ values to non-integer values of $Z$ but instead used extrapolations of $\Delta$ only, which show no such irregularities.

The $3 s-3 p$ transitions are the best candidates for a comparison of the theoretical corrected values with extrapolated values, obtained from Edlen's very accurate empirical formula [6], which reads:

$$
\begin{aligned}
& \sigma\left(\mathrm{cm}^{-1}\right)=16,213.3 \xi+13,832.1-60,077(\xi-3.53)^{-1} \\
& +0.8(\xi+5)^{3}
\end{aligned}
$$

where $\xi=$ nuclear charge - number of electrons +1 . We used this formula for the $3 s-3 p$ transitions and give the results in the third part of column 4 , which are the predicted values. The agreement between all three values in column 4 is better than $10 \AA$ for all $Z$ (with the exception of $Z=101 / 3$ ). This good agreement between the two results confirms the reliability of the numbers obtained. It also indicates that the Lagrange extrapolation, used for the deviation $\delta$ and $\Delta$, is a reasonable extrapolation. The accuracy of $\delta$ is expected to be less than $2 \%$, which leads to an uncertainty of $\pm 10 \AA$ for the $3 s-3 p$ transitions. For $Z=101 / 3$ case the error may be larger, but since it is not clear how to estimate the error here, the transition wavelengths for $Z=101 / 3$ have to be accepted with caution. They are given here only to demonstrate the general applicability of the methods employed. For $Z=111 / 3,112 / 3$ cases interpolation rather than extrapolation is required to obtain the deviations $\delta$. The values, therefore, are expected to be much more accurate than those for $Z=102 / 3$ and especially for
$Z=101 / 3$ case. The difference between the values obtained here and those obtained from Edlen's formula are only a few $\AA$.

For the $3 s-4 p, 3 p-3 d$, and $3 p-4 s$ transitions comparison with a value, extrapolated with a formula comparable to Edlen's formula (1), was not possible because of the difficulties in obtaining an extrapolation formula of the kind used for the $3 s-3 p$ transitions by us. Most of the interpolation formulae result in values lower than the corrected ones, i.e. by as much as $20 \%$. Therefore, only the corrected values are listed.

\section{Discussion}

The predicted values in Tables $2 \mathrm{a}-2 \mathrm{e}$ show clearly that the shifts in the transition wavelengths for Na-like systems with $Z=11 \pm 1 / 3, \pm 2 / 3$ are substantial. The most uncertain values are those for a nucleus with charge $Z=101 / 3$. This atom is nearly a negative ion. The outermost electron is almost unbound and thus the transition energies are shifted to very small energies, i.e. large wavelengths. The transition with the smallest wavelength, discussed in Table 2, is the $3 s-4 p$ transition with over $17,000 \AA$. This is much too far into the $I R$ region to be detectable in an easy way. To make a simple presentation, from an experimental point of view, we summarized all transition energies, within the region of visible light, from Table 2, for all cases in Fig. 1. In addition we included the main lines for neutral $\mathrm{Na}$. The most dominant, of course, is the famous $\mathrm{Na}-\mathrm{D}$ line at $5,890 \AA$. In this region of visible light we find one transition for the $102 / 3$ atom, three for the $111 / 3$ atom, and one for the $112 / 3$ atom. Only one transition around $5,150 \AA$ is so near a normal $\mathrm{Na}$ line that it probably cannot be used to detect a quark atom. All other transitions are so far away from each other (including the maximum estimated error of $\pm 10 \AA$ ) that the detection of even a single atom amongst as many as $10^{19}$ other atoms may be possible by use of tuneable dye lasers. The most likely candidate for a quark atom is $102 / 3$. It probably has a line at $5,955 \AA$ which should be an easily accessible region for this method.



Fig. 1. Spectral transitions of neutral $\mathrm{Na}(Z=11)$ and $\mathrm{Na}$ quark atoms with charges $Z=11 \pm 1 / 3, \pm 2 / 3$ in the region of optical wavelengths 
In conclusion we might say that a number of possible transitions exist in the region of visible light which might be used to detect atoms with additional charges $\pm 1 / 3$ and $\pm 2 / 3$.

The authors wish to thank the International Centre for Theoretical Physics, Kernforschungszentrum Karlsruhe, and Pakistan Atomic Energy Commission for financial support. K.R. whishes to thank the National Science Foundation for a travel grant, and P. Trower for the warm hospitality at Virginia Techn. K.R. also wishes to thank A. Winther for a stay at the Niels Bohr Institute where this work was completed.

\section{References}

1. LaRue, G.S., Fairbank, W.M., Hebard, A.F.: Phys. Rev. Lett. 38 , $1011(1977)$

LaRue, G.S., Fairbank, W.M., Phillips, J.D.: Phys. Rev. Lett. 42, 142 (1979)

LaRue, G.S., Phillips, J.D., Fairbank, W.M.: Phys. Rev. Lett. 46, 967 (1981)

2. Fairbank, W.M. jr., She, C.Y.: Optic News, Spring 1980

Fairbank, W.M. jr., Hänsch, T.W., Schawlow, A.L.: J. Opt. Soc. Am. 65, 199 (1965)

Hurst, G.S., Nayfeh, M.H., Young, J.P.: Appl. Phys. Lett. 30, 229 (1977)
Fairbank, W.M. jr.: Near zero; New frontiers in physics conference. Stanford University (1982)

3. Massey, H.: Negative ions. Cambridge: Cambridge University Press 1976

4. Lindgren, I., Rosén, A.: Case studies in atomic physics. Phys. Rep. 4, 93 (1974)

Grant, I.P.: Adv. Phys. 19, 747 (1970)

5. Desclaux, J.P.: Comp. Phys. Commun. 9, 31 (1975)

6. Edlen, B.: Handbuch der Physik. Spectroscopy I. Vol. XXVII. Berlin, Heidelberg, New York: Springer;

Edlen, B.: Phys. Scr, 17, 565 (1978)

7. Bashkin, S., Stoner, J.O.: Atomic energy levels and grotian diagrams. Amsterdam, Oxford, New York: North Holland 19

\section{Khalid Rashid}

Nuclear Physics Division

Pakistan Institute of

Nuclear Science and Technology

P.O. Nilore

Rawalpind

Pakistan

Burkhard Fricke

Fachbereich Physik

Theoretische Physik

Gesamthochschule Kassel

Heinrich-Plett-Strasse 40

D-3500 Kassel-Oberzwehren

Federal Republic of Germany 